\title{
Wie weit ist die Schweiz mit der Tabakprävention?
}

\author{
Als Thema des Welttags ohne Tabak 2011 hat die Weltgesundheitsorganisation WHO \\ die internationale Rahmenkonvention über die Tabakkontrolle bestimmt. Die Schweiz \\ hat die Konvention 2004 unterzeichnet, bisher aber nicht ratifiziert. Hindernis für \\ eine Ratifizierung ist vor allem das fehlende Verbot von Werbung, Promotion und \\ Sponsoring für Tabakprodukte.
}

\author{
Thomas Beutler ${ }^{a}$, \\ Nicolas Broccard ${ }^{b}$, \\ Verena El Fehri ${ }^{a}$ \\ a Arbeitsgemeinschaft \\ Tabakprävention Schweiz, \\ Bern \\ b Wissenschaftsjournalist, Bern
}

Korrespondenz:

Verena El Fehri Geschäftsführerin Arbeitsgemeinschaft Tabakprävention Schweiz AT Haslerstrasse 30

CH-3008 Bern

Tel. 0315991020

Fax 0315991035

info@at-schweiz.ch

www.at-schweiz.ch
Die WHO-Rahmenkonvention über die Tabakkontrolle enthält die Grundsätze, die im 21. Jahrhundert die globale Verbreitung des Tabaks und der Tabakwaren lenken sollen. Die Konvention trat 2005 in Kraft. Bis jetzt haben sie 172 Mitgliedstaaten der Weltgesundheitsorganisation ratifiziert. Wie die Grundsätze umzusetzen sind, wird in den Richtlinien zu einzelnen Artikeln festgelegt. Bei den Verhandlungen hat die Schweiz bloss Beobachterstatus. Die Vertragsparteien haben bereits mehrere Richtlinien verabschiedet, unter anderem zu Werbung, Promotion und Sponsoring (Art. 13), Schutz vor Passivrauchen (Art. 8), Verpackung und Etikettierung (Art. 11), Schutz vor den Interessen der Tabakindustrie (Art. 5.3), Information (Art. 12) und Verminderung der Nachfrage (Art. 14).

\section{Werbung, Promotion, Sponsoring}

Die (von der Tabakindustrie unabhängige) Forschung hat eindeutige Resultate ergeben:

- Werbung, Verkaufsförderung und Sponsoring für Tabakwaren erhöhen den Tabakkonsum.

- Ein umfassendes Verbot derartiger Aktivitäten senkt den Konsum.

Die entsprechenden Richtlinien verlangen ohne Ausnahme ein Verbot aller Aktivitäten, die direkt oder indirekt den Tabakkonsum fördern. In der Schweiz ist auf nationaler Ebene nur Werbung in Radio und Fernsehen sowie Werbung, die sich speziell an Jugendliche unter 18 Jahren richtet, untersagt. Hinzu kommen zusätzliche Einschränkungen in 14 Kantonen. Solche Verbote in Teilbereichen haben meist zur Folge, dass die Tabakfirmen ihre Werbeaktivitäten auf andere Teilbereiche verlagern. Verkaufsförderung und Sponsoring sind in der Schweiz grösstenteils unbeschränkt möglich.

Einzig ein Verbot, das alle Bereiche einschliesst, trägt wirksam zur Senkung des Tabakkonsums bei. In Europa kennen bereits Norwegen, Irland, Grossbritannien (ab Oktober 2011) und Finnland (ab Januar 2012) ein umfassendes Verbot, das auch für Werbung und Auslage an den Verkaufsstellen gilt.

\section{Passivrauchen}

Jährlich sterben in der Schweiz ungefähr 1000 Personen vorzeitig an den Folgen des Passivrauchens. Um

\section{Zusammenfassung}

Die WHO-Rahmenkonvention über die Tabakkontrolle enthält die Grundsätze, die im 21. Jahrhundert die globale Verbreitung des Tabaks und der Tabakwaren lenken sollen. $\mathrm{Zu}$ den wirksamen als auch kostengünstigen Massnahmen, um den Tabakkonsum zu senken, zählen unter anderem ein umfassendes Verbot aller Werbeformen für Tabak und Gesetze für rauchfreie Lebenswelten. Die Schweiz hat die Konvention 2004 unterzeichnet, bisher aber nicht ratifiziert. Hindernis für eine Ratifizierung ist vor allem das fehlende Verbot von Werbung, Promotion und Sponsoring für Tabakprodukte. Verkaufsförderung und Sponsoring sind in der Schweiz grösstenteils unbeschränkt möglich. Auch hinsichtlich des Schutzes vor Passivrauchen enthält das Bundesgesetz zum Schutz vor Passivrauchen grosse Lücken. Für einen vollständigen Schutz reichte im Mai 2010 die Allianz «Schutz vor Passivrauchen», in der auch die Verbindung der Schweizer Ärztinnen und Ärzte FMH vertreten ist, eine eidgenössische Volksinitiative ein.

Vom 19. bis 20. September 2011 findet in New York der UNO-Gipfel zu nicht übertragbaren Krankheiten statt. In einem Aufruf in «The Lancet» haben über vierzig Expertinnen und Experten aus der ganzen Welt die Tabakkontrolle zuoberst auf die Prioritätenliste des UNO-Gipfels gesetzt. Als Ziel für 2040 fordern sie, dass weniger als 5 Prozent aller Menschen Tabak konsumieren.

Krankheit, Invalidität und Tod durch Passivrauchen zu senken, fordern die Richtlinien strenge Massnahmen zum Schutz vor Passivrauchen: 
- Nur hundertprozentig rauchfreie Innenräume ermöglichen einen wirksamen Schutz. Andere Massnahmen wie Lüftungsanlagen oder Rauchzonen sind erwiesenermassen unwirksam.

- Alle Menschen sind vor Passivrauchen zu schützen. Alle Arbeitsplätze in Innenräumen und alle öffentlich zugänglichen Innenräume müssen rauchfrei sein.

- Für den Schutz vor Passivrauchen sind Gesetze notwendig. Freiwillige Selbstbeschränkungen, wie von der Tabakindustrie bevorzugt, haben sich wiederholt als unwirksam herausgestellt.

In Europa schreiben 18 Länder einen strengen Schutz vor Passivrauchen vor. Die Schweiz gehört noch nicht zu diesen Ländern. Das Bundesgesetz zum Schutz vor Passivrauchen erlaubt das Rauchen an Einzelarbeitsplätzen und gestattet in der Gastronomie Rauchräume mit Bedienung und Rauchbetriebe. Verschiedene Kantone haben weitergehende Vorschriften als der Bund beschlossen.

\section{Eidgenössische Volksinitiative}

Die Allianz «Schutz vor Passivrauchen», in der auch die Verbindung der Schweizer Ärztinnen und Ärzte FMH vertreten ist, reichte im Mai 2010 eine Volksinitiative ein. Diese will die Lücken des Bundesgesetzes schliessen:

- Alle Arbeitsplätze in Innenräumen sind rauchfrei.

- Ebenso sind in der Regel öffentlich zugängliche Räume inklusive Restaurations- und Hotelbetriebe rauchfrei.

- In der ganzen Schweiz soll die gleiche Regelung gelten. So schafft die Initiative einheitliche Bedingungen für alle Gastwirtinnen und Gastwirte sowie alle Gäste.

Im November 2010 entschied der Bundesrat, die Volksinitiative ohne Gegenvorschlag abzulehnen. In der Botschaft an das Parlament von März 2011 schreibt er, «die derzeitige Gesetzgebung reicht aus, um die Gesundheit der Arbeitnehmerinnen und Arbeitnehmer und der Bevölkerung zu schützen». Dies ist unzutreffend, besonders in bedienten Rauchräumen und Rauchbetrieben sind die Beschäftigten dem Passivrauchen schutzlos ausgeliefert. Zudem will der Bundesrat zuerst «Erfahrungen sammeln» und daraus «Lehren ziehen». Das ist unnötig. Die Auswertungen der Erfahrungen aus unzähligen Ländern laufen stets auf dieselbe Schlussfolgerung hinaus: Allein in vollständig rauchfreien Innenräumen ist ein wirksamer Schutz vor Passivrauchen garantiert.

\section{Rauchstopp-Wettbewerb 2011}

Die effizienteste Massnahme, um die durch das Rauchen verursachte Sterblichkeit zu senken, ist die Erhöhung der Anzahl von Rauchern, die den Tabakkonsum aufgeben. Dazu führt das Nationale Rauchstopp-Programm jährlich zum Welttag ohne Tabak den Rauchstopp-Wettbewerb durch. Teilnehmen können alle Raucherinnen und Raucher, die vom 4. Juni bis zum 4. Juli 2011 eine Rauchpause einschalten. Anmeldung und Bestellung von Unterlagen bei der Arbeitsgemeinschaft Tabakprävention auf www.at-schweiz.ch oder unter Nummer 0315991020.

\section{Verpackung}

Gerade Raucherinnen und Raucher unterschätzen häufig die Gesundheitsrisiken des Tabakkonsums. Unmissverständliche Warnhinweise auf Tabakpackungen erhöhen nachweislich die Aufmerksamkeit für die Risiken und die Bereitschaft zum Rauchstopp.

Die in der Schweiz vorgeschriebenen Warnhinweise mit Bild und Text entsprechen der EU-Richtlinie von 2001 über Herstellung, Aufmachung und Verkauf von Tabakprodukten. Die Richtlinie wird zurzeit überarbeitet. In der Vernehmlassung haben Nichtregierungsorganisationen sich unter anderem eingesetzt für Einheitspackungen ohne Werbung. Als erstes Land plant Australien Einheitspackungen auf Juli 2012 einzuführen.

\section{Interessen der Tabakindustrie}

Jede Art des Tabakkonsums macht süchtig und verursacht Krankheit und Tod. Wirksame Massnahmen zur Tabakprävention und -kontrolle lösen einen Rückgang des Tabakkonsums aus. Aber die Tabakindustrie lehnt wirksame Präventionsmassnahmen ab. Stattdessen ist sie bestrebt, möglichst viele Tabakwaren zu verkaufen und einen möglichst hohen Gewinn zu erwirtschaften.

Folglich besteht zwischen den Interessen von Public Health und den Interessen der Tabakindustrie ein grundlegender und unversöhnlicher Konflikt. Bei der Ratifizierung der Rahmenkonvention muss sich deshalb jede Vertragspartei verpflichten, die Massnahmen zur Tabakkontrolle vor den Interessen der Tabakindustrie zu schützen.

\section{UNO-Gipfel über nicht übertragbare Krank- heiten}

Weltweit sterben heute an den Folgen des Tabakkonsums, gemäss Schätzungen der WHO, jährlich fünf Millionen Menschen. Wird die Tabakepidemie nicht gestoppt, werden es im Jahr 2030 acht Millionen Menschen sein.

Vom 19. bis 20. September 2011 findet in New York der UNO-Gipfel über nicht übertragbare Krankheiten statt. In einem Aufruf in «The Lancet» haben über vierzig Expertinnen und Experten aus der ganzen Welt die Tabakkontrolle zuoberst auf die Prioritätenliste des UNO-Gipfels gesetzt. Nötig sei die volle Umsetzung der WHO-Rahmenkonvention [1]. Als Ziel für 2040 wird im Aufruf gefordert, dass weniger als 5 Prozent aller Menschen Tabak konsumieren; in der Schweiz rauchen heute mehr als 25 Prozent der Bevölkerung. Zu den wirksamen als auch kostengünstigen Massnahmen, um den Tabakkonsum zu senken, zählen die Expertinnen und Experten hohe Tabaksteuern, gesetzlich vorgeschriebene Warnhinweise, Gesetze für rauchfreie Lebenswelten und ein umfassendes Verbot aller Werbeformen für Tabak.

1 Beaglehole R, Bonita R, Horton R, Adams C, Alleyne G, Asaria P. Priority actions for the non-communicable disease crisis. The Lancet. 2011; 377:1438-47.

Mehr zur WHO-Rahmenkonvention siehe www.who. int/fctc/en 